\title{
EI TERCE en poblaciones vulnerables de Panamá
}

\section{The TERCE in vulnerable populations of Panama}

\author{
Autores: Daysi Jackson ${ }^{1 *}$, Rosa Arrocha ${ }^{2} \&$ Jaime Estrella $^{3}$ \\ ${ }^{1}$ Docente, Colegio San Carlos, Ministerio de Educación. Panamá. \\ (D https://orcid.org/0000-0003-2880-0440 \\ ${ }^{2}$ Docente, Departamento de Estadística, Facultad de Economía, Universidad de Panamá. Panamá. \\ (D.https://orcid.org/0000-0003-1341-5259 \\ ${ }^{3}$ Investigador independiente, Especialista en Gestión de Investigación. Panamá. \\ (Dhttps://orcid.org/0000-0002-8020-2184
}

* Autora por correspondencia: Daysi Jackson, daysinoemi_jacksontapia@ hotmail.com

Recibido: 09 de septiembre de 2020

Aceptado: 20 de julio de 2021

\begin{abstract}
Resumen
Este estudio tuvo como objetivo identificar las escuelas oficiales de Panamá que obtuvieron niveles superiores al promedio nacional en las pruebas de lectura, matemática y ciencias en tercero y sexto grado, y que se encontraban en contextos desfavorables. Este artículo muestra el análisis realizado mediante una investigación cuantitativa empleando los datos del Tercer Estudio Regional Comparativo Explicativo (TERCE), llevado a cabo por el LLECE de la UNESCO (año 2013). Las 137 escuelas oficiales se agruparon mediante el método de conglomerados de $\mathrm{K}$ medias, en cuatro conglomerados, según el nivel socioeconómico y cultural. En el conglomerado con los índices promedios más severos, esto es -1.44 para estudiantes de sexto y -1.22 para tercer grado, no se encontró ninguna escuela con promedios superiores al promedio nacional. En el conglomerado con índice de -0.62 para sexto grado y -0.60 para tercer grado, que fue el segundo en cuanto a la severidad de condiciones desfavorables, se encontraron tres escuelas con promedios superiores al nacional, en todas las áreas curriculares evaluadas. Los conglomerados reflejaron evidencia de diferencias debido a la condición socioeconómica de las escuelas, lo cual permitirá formular recomendaciones de política nacional educativa para Panamá.
\end{abstract}

Palabras clave: análisis de conglomerados, contexto desfavorable, índice socioeconómico, Panamá, poblaciones vulnerables, TERCE.

\begin{abstract}
This study aimed to identify official schools in Panama that obtained averages higher than the national average in the tests of reading, mathematics, and science in third and sixth grade, which were in unfavorable contexts. This article shows the analysis made through quantitative research, using data from the Third Explanatory Regional Comparative Study (TERCE, after its acronym in Spanish), conducted by the LLECE of the UNESCO (2013). According to the socioeconomic and cultural level, the 137 official schools were grouped in four clusters through the $\mathrm{K}$ media conglomerate method. In the cluster with the most severe averages, i.e., -1.44 for sixth grade and -1.22 for third grade, respectively, no schools with averages above the national average were
\end{abstract}


found. In the cluster with an index of -0.62 for sixth grade and -0.60 for third grade (which was the second cluster in terms of severity of unfavorable conditions), three schools with averages higher than the national value were found in all evaluated curricular areas. Clusters obtained showed differences due to socioeconomic conditions among schools, allowing the formulation of recommendations for national educative policy in Panama.

Keywords: cluster analysis, Panama, socioeconomic index, TERCE, unfavorable context, vulnerable populations.

\section{Introducción}

Panamá ha participado, desde el año 2005 en adelante, en diversas pruebas de logros educativos ${ }^{1}$ a gran escala a nivel nacional y regional. Su participación ha sido, por ejemplo, en el SINECA ${ }^{2}$ 2005, SERCE $^{3} 2006$, SINECA 2008, PISA ${ }^{4}$ 2009, TERCE 2013, las Pruebas CRECER 2016, 2017 y 2018, así como en PISA 2018 y ERCE 2019. Sin embargo, ante la gran cantidad de evaluaciones realizadas a lo largo de estos últimos 15 años, los estudios derivados de las mismas son escasos en Panamá.

Un estudio cualitativo ${ }^{5}$ realizado por Jackson et al. (2017), muestra que, en escuelas panameñas de nivel socioeconómico bajo, el involucramiento de la familia, la vocación del maestro y el deseo de aprender por parte del estudiante son factores determinantes en un buen rendimiento.

Chaparro et al. (2016) identificaron perfiles de estudiantes de secundaria, basados en variables de rendimiento académico y nivel social y cultural de las familias. Este estudio permitió concluir que las variables familiares analizadas permiten configurar perfiles estudiantiles que se asocian con el rendimiento académico.

Por otra parte, el estudio "Efecto del nivel socioeconómico en el rendimiento de los estudiantes peruanos: un balance de los últimos 15 años" (León y Collahua, 2016), realizó un metaanálisis de la relación entre el nivel socioeconómico de las familias y el rendimiento académico de los estudiantes peruanos, en los años 2000 hasta 2014. Este estudio dio como resultado que el nivel socioeconómico resulta ser una variable importante para explicar el rendimiento de los estudiantes peruanos, ya sea en el ámbito individual o escolar.

Murillo y Hernández (2011) centraron su investigación en los efectos escolares sobre variables socioafectivas, tales como: autoconcepto, disciplina, convivencia social y satisfacción con la escuela. Entre los resultados obtenidos es posible mencionar que los estudiantes de grupos indígenas tienen menor autoconcepto y satisfacción por la escuela, no así estudiantes de un nivel-socioeconómico alto, quienes tienen un adecuado autoconcepto, adecuado comportamiento, se adaptan mejor y se sienten satisfechos con la escuela. Otro resultado interesante de este estudio fue el hallazgo de que la escuela tiene una importancia muy pequeña en el desarrollo de productos socioafectivos.

Entre los numerosos estudios realizados en México por el Instituto Nacional para la Evaluación de la Educación (INEE, 2017), resalta el denominado "La educación para poblaciones en contextos vulnerables", en que se indica que la relación del entorno de los alumnos y los materiales de las escuelas interactúan y se refuerzan

${ }^{1}$ En Panamá, las pruebas de logros son de carácter formativo y el ente responsable de aplicarlas es la Dirección Nacional de Evaluación Educativa (DNEE) del Ministerio de Educación (MEDUCA); su fundamento legal es el Decreto Ejecutivo No. 878 de septiembre 27 de 2016.

2 SINECA: Sistema Nacional de Evaluación de la Calidad, estudio llevado a cabo por la Dirección Nacional de Evaluación Educativa del MEDUCA, Panamá.

3 SERCE: Segundo Estudio Regional Comparativo y Explicativo, llevado a cabo por el Laboratorio Latinoamericano de la Evaluación de la Calidad de la Educación (LLECE, Santiago de Chile).

${ }^{4}$ PISA: Programme for International Student Assessment, estudio llevado a cabo por la Organización para la Cooperación y el Desarrollo Económicos (OCDE).

${ }^{5}$ Este estudio fue realizado por Jackson, Estrella y Rodríguez; es derivado de la evaluación del TERCE en escuelas panameñas en contextos desfavorables. El estudio se realizó con financiamiento de la Secretaría Nacional de Ciencia, Tecnología e Innovación (SENACYT, Panamá). 
entre sí. Los hallazgos presentados por este estudio tienen relación con otros estudios similares. Una de las conclusiones de este estudio del INEE fue que para ofrecer una educación de calidad es necesario preparar mejor a los docentes para hacerle frente a situaciones adversas y desafiantes en sus aulas, que están compuestas con grupos de la población más desfavorecida, además de mejores instalaciones, equipos y materiales pertinentes a las necesidades y prioridades de estos grupos.

Con estos antecedentes, este estudio se enfocó en el TERCE en Panamá. Este estudio regional comparativo aportó información sobre la calidad de la educación; no sólo aplicó pruebas para medir logros de aprendizaje, sino también cuestionarios para comprender el contexto y entender las circunstancias bajo las cuales el aprendizaje ocurrió en las áreas evaluadas. Uno de los aportes destacados del TERCE, que lo distingue de otros estudios internacionales, es que se realizó un análisis de los factores asociados al logro del aprendizaje, con el objetivo de entender bajo qué circunstancias ocurrió el aprendizaje en los estudiantes. Para el caso específico de Panamá, el TERCE significó tener información sobre aspectos socioeconómicos de las familias, los factores relacionados a la praxis del maestro, la gestión del director y el clima del aula, entre otras variables que afectan el aprendizaje. Además, de lo anteriormente mencionado, el presente estudio permite disponer de una "radiografía" de lo que está ocurriendo en educación, con el fin de potenciar acciones concretas y efectivas a nivel de aula, escuela y del Ministerio de Educación, para lograr una educación de calidad en Panamá.

El objetivo de este estudio fue identificar escuelas oficiales (públicas) de Panamá que obtuvieron un puntaje promedio en logros de aprendizaje en las pruebas TERCE, por arriba del promedio nacional en las pruebas de lectura, matemática y ciencias, y que pertenecen a un nivel socioeconómico desfavorable, a fin de caracterizar el contexto socioeconómico al que pertenecen.

\section{Materiales y métodos}

\section{El estudio TERCE y la participación de Panamá}

EL TERCE es una evaluación que se implementó en el año 2013, en la cual participaron 15 países de la región de Latinoamérica, más el estado de Nuevo León (México). Este estudio entregó información sobre la eficacia escolar y dio cuenta de las brechas en los niveles de logros de aprendizajes entre estudiantes de diferentes contextos socioculturales, con el fin de que los países puedan tener un panorama del sistema educativo que les permita reflexionar sobre lo que se hace en las escuelas y, de este modo, crear políticas públicas que vayan hacia el mejoramiento de la calidad educativa.

Su evaluación consistió en medir los logros de aprendizaje en estudiantes de tercer y sexto grado en lenguaje (lectura y escritura), matemática y ciencias. Además, el TERCE identificó factores asociados a los logros de aprendizaje (UNESCO, 2019). Para tal propósito, dicha evaluación regional utilizó dos instrumentos de recolección de datos: pruebas de evaluación de aprendizaje y cuestionarios de contexto.

Panamá participó en el TERCE con una muestra representativa de 184 escuelas, de las cuales 137 eran del sector oficial (públicas) y 47 del sector particular. Esta distribución se aprecia en la Tabla 1. Dicha tabla muestra las escuelas efectivas que participaron del estudio que se describe a continuación. En el sistema educativo panameño, el mayor número de escuelas primarias está en las áreas rurales, pero la mayor cantidad de población estudiantil se concentra en las escuelas urbanas. 
Tabla 1.

Tipos de escuela y muestra experimental del TERCE, por tipo de dependencia según área geográfica, en Panamá.

\begin{tabular}{lccc}
\hline Tipo de escuela & Públicas & Particulares & Total \\
\hline Urbanas & 26 & 46 & 72 \\
Rurales & 111 & 1 & 112 \\
Total de escuelas & $\mathbf{1 3 7}$ & $\mathbf{4 7}$ & $\mathbf{1 8 4}$ \\
\hline
\end{tabular}

\section{Tipo de estudio y análisis de conglomerados}

El tipo de estudio fue cuantitativo, no experimental en campo y con un componente documental. Esto, en vista de que se revisaron los documentos de contexto del TERCE, para luego analizar la base de datos generada por esta evaluación, seleccionando los datos de Panamá únicamente. Este estudio también fue de carácter diagnóstico, descriptivo y explicativo, para determinar los resultados relativos a los logros y retos en educación a raíz de la aplicación del TERCE en Panamá.

Para identificar escuelas con promedios por encima de la media nacional, pero en contextos desfavorables, se realizó un análisis de conglomerados. La fuente de información para este análisis fue, en primer lugar, el documento "Informe de resultados TERCE: logros de aprendizaje" compilado por la UNESCO, en el contexto del LLECE (2015) ${ }^{6}$. Adicionalmente, para el análisis estadístico y el respectivo tratamiento de las variables, se recurrió a la base de datos completa disponible para descarga desde el repositorio del Laboratorio Latinoamericano de Evaluación de la Calidad de la Educación (LLECE), de la Oficina Regional de Educación para América Latina y el Caribe (de la UNESCO, en Santiago de Chile) ${ }^{7}$.

El análisis de conglomerados aplicado en este estudio se enfocó como una técnica estadística para clasificar los datos y observaciones en grupos. A los grupos obtenidos se les llamó conglomerados (o clusters). Los grupos que se formaron por el análisis estadístico son muy similares con respecto a las características de estudio dentro del grupo, pero muy diferentes entre grupos respecto a esas características. Para evaluar la precisión de que un par de observaciones se parecen entre sí, se utilizó el indicador de distancia o similitud. En este caso, las observaciones fueron las escuelas de Panamá; para este estudio fueron agrupadas por el método de conglomerados de K medias. Dicho método se basa en la distancia existente entre ellas. En este marco, el procesamiento de los datos fue realizado con el software SPSS versión 22 (IBM Corp., 2013).

La variable de interés para conformar los conglomerados fue el índice de nivel socioeconómico. El índice de nivel socioeconómico es una medida que se tomó de la base de datos y procedimientos definidos por el LLECE (UNESCO 2015 y 2019). Su cálculo se basó en las respuestas que los padres y madres de familia respondieron a un cuestionario con respecto al material de construcción de la vivienda, bienes y servicios con los que se cuenta en el hogar, nivel educativo alcanzado por la madre, ocupación e ingreso total del hogar y cantidad de libros disponibles en el hogar. Estos valores se expresaron como porcentajes, tal como se presenta en la siguiente sección. Lo que caracteriza a este método es que en cada conglomerado las escuelas son muy parecidas entre sí respecto al índice de nivel socioeconómico de las familias de los estudiantes de tercero y sexto grado de las escuelas en Panamá. Pero, en los diferentes conglomerados son muy diferentes respecto a las variables que se consideraron, para identificar los patrones que los hacen distinguibles del resto.

Adicionalmente, se analizó los puntajes promedios por conglomerados, calculando los intervalos de confianza, con la finalidad de detectar las diferencias significativas en el rendimiento entre los diferentes conglomerados.

\footnotetext{
${ }^{6}$ El informe está disponible para descarga en texto completo desde la Biblioteca Digital UNESDOC (de la UNESCO) en el enlace: https://unesdoc.unesco.org/ark:/48223/pf0000243532.

${ }^{7}$ La información detallada sobre este repositorio, así como la base de datos completa, están disponibles en el enlace: https://leceunesco.org/explora/panama/desempeno-y-resultados-orientaciones/
} 
A través de intervalos de confianza se describió el índice de nivel socioeconómico para cada conglomerado. Mediante gráficas se analizaron, para cada conglomerado, las variables de las familias de los estudiantes como son: educación del padre y madre, trabajo pagado para el padre y madre, servicios básicos de viviendas y expectativas de los padres en cuanto al nivel educativo más alto que alcanzarán sus hijos.

Finalmente, por medio de gráficas se caracterizaron las escuelas por conglomerados, con respecto a las variables denominadas "accede al puesto de director" y "facilidades con las que cuentan las escuelas". Tal como se verá más adelante, en este estudio se muestra con énfasis las características del conglomerado C, que es aquel en donde se encontraron las escuelas en contextos desfavorables, con promedio por arriba del promedio nacional en tercero y sexto grado (el criterio de estudio anteriormente mencionado), pero también se hace referencia a aspectos importantes de los restantes conglomerados.

Este estudio no tuvo una fase de intervención en campo; sin embargo, se desarrolló en apego a la ética en la investigación. En este sentido, se coordinó oportunamente con el LLECE para el uso de la base de datos (pese a ser datos en acceso abierto y dominio público); y, se mantuvo total confidencialidad sobre los nombres de las escuelas en el análisis de conglomerados. Finalmente, a los conglomerados se les asignó las letras A, B, C y $\mathrm{D}$, de manera arbitraria, para fines de describirlos en la sección que se presenta a continuación.

\section{RESULTADOS}

El análisis por conglomerados se realizó en 128 escuelas oficiales $^{8}$, para tercero y sextos grados en la República de Panamá, que son las que participaron efectivamente en el TERCE. El índice socioeconómico para los estudiantes de sexto grado varió de -2.18 a 0.89 y el índice promedio fue de - 0.5467. En los estudiantes de tercer grado varió de - 1.51 a 0.68 y el índice promedio fue de - 0.4878 . Como el rango de variación del índice en tercer grado es menor que el de sexto grado (la diferencia entre el índice mayor 0.68 y el índice menor 1.51), esto implicó que las familias de los estudiantes de tercer grado son más similares estadísticamente en cuanto al nivel socioeconómico que las familias de los estudiantes de sexto grado.

\section{Análisis de conglomerados}

A través del análisis de conglomerados se identificaron cuatro grupos. En la Tabla 2 se muestran los cuatro conglomerados obtenidos: el conglomerado A es el de nivel socioeconómico más alto (o de mejores condiciones); en segundo lugar, está el conglomerado B; en tercer lugar, el conglomerado C; y, en cuarto lugar, el conglomerado D.

Tabla 2.

Número de escuelas públicas e índice promedio de nivel socioeconómico por grado según el análisis de conglomerados, en el TERCE Panamá.

\begin{tabular}{lccc}
\hline Conglomerado & $\begin{array}{c}\text { Número de } \\
\text { escuelas }\end{array}$ & \multicolumn{2}{c}{ Índice promedio (por grado) } \\
\cline { 2 - 4 } & & Sexto & Tercero \\
\hline A & 29 & 0.24 & 0.13 \\
& 35 & -0.27 & -0.20 \\
B & 30 & -0.62 & -0.60 \\
C & 34 & -1.44 & -1.22 \\
D & $\mathbf{1 2 8}$ & $\mathbf{- 0 . 5 5}$ & $\mathbf{- 0 . 4 9}$ \\
\hline
\end{tabular}

\footnotetext{
${ }^{8}$ El número final de escuelas que participaron efectivamente en el TERCE en Panamá varió, debido a limitaciones en la aplicación de la prueba en campo.
} 


\section{Rendimiento de las escuelas según conglomerados}

Para analizar en rendimiento promedio de las escuelas según las características socioeconómicas del conglomerado al que pertenecen, se cuantificó el porcentaje de escuelas que obtuvieron puntaje promedio en las diferentes pruebas por arriba de la media nacional. A los estudiantes de sexto grado se les aplicó pruebas de lectura, matemática y ciencias naturales.

\section{Rendimiento de las escuelas según conglomerados para tercer grado}

A los estudiantes de tercer grado se les aplicó pruebas de lectura y matemática solamente (es decir, dos pruebas). En la Tabla 3 se presenta la cantidad de escuelas públicas de Panamá según el número de pruebas, que obtuvieron puntajes por arriba de la media nacional, según el conglomerado donde fueron clasificadas. Se destacan algunos aspectos que se describen a continuación.

\section{Tabla 3.}

Número de escuelas públicas según la cantidad de pruebas de tercer grado donde obtuvieron un puntaje promedio por arriba de la media nacional.

\begin{tabular}{lcccc}
\hline Conglomerado & Total & \multicolumn{3}{c}{ Número de escuelas con promedio por arriba del promedio } \\
& & & nacional & \\
\cline { 3 - 5 } & & 2 pruebas & 1 prueba & 0 pruebas \\
\hline A & 29 & 19 & 3 & 7 \\
B & 35 & 5 & 10 & 10 \\
C & 30 & 5 & 14 & 11 \\
D & 34 & 4 & 2 & 28 \\
\hline Total de escuelas & $\mathbf{1 2 8}$ & $\mathbf{4 3}$ & $\mathbf{2 9}$ & $\mathbf{5 6}$ \\
\hline
\end{tabular}

El conglomerado A, caracterizado por el índice de nivel socioeconómico más alto, está conformado por 29 escuelas, de las cuales $19(66 \%)$ obtuvieron un promedio por arriba de la media nacional en las dos pruebas aplicadas y 7 escuelas (24\%) tuvieron un promedio por debajo de la media nacional en las dos pruebas aplicadas.

El conglomerado B, que está en segundo lugar en cuanto a las condiciones socioeconómicas, está conformado por 35 escuelas. Se observa que 15 escuelas (43\%) obtuvieron promedio por arriba de la media nacional en las dos pruebas y 10 escuelas (29\%) obtuvieron puntajes por debajo de la media en las dos pruebas.

El conglomerado C, que está en tercer lugar en cuanto a nivel socioeconómico, está conformado por 30 escuelas (Tabla 3). En este caso, cinco escuelas (el 17\%) obtuvieron puntajes por arriba de la media nacional, mientras que 11 escuelas (el 37\%) obtuvieron un promedio por debajo de la media nacional.

El conglomerado D (que tiene las condiciones socioeconómicas más desfavorecidas) lo conforman 34 escuelas; de éstas, cuatro escuelas (12\%) obtuvieron promedios por arriba de la media nacional y 28 escuelas (un $82 \%$ ) obtuvieron promedio por debajo de la media nacional en las dos pruebas.

\section{Rendimiento de las escuelas según conglomerados para sexto grado}

En la Tabla 4 se muestra la cantidad de pruebas con puntajes sobre la media nacional obtenida por las escuelas públicas de Panamá en el TERCE. Algunos aspectos para destacar luego de realizado el análisis estadístico se presentan a continuación. 
Tabla 4.

Número de escuelas públicas según la cantidad de pruebas de sexto grado donde obtuvieron un puntaje promedio por arriba de la media nacional.

\begin{tabular}{lccccc}
\hline Conglomerado & Total & \multicolumn{5}{c}{ Número de escuelas con promedio por arriba del promedio } \\
& & \multicolumn{4}{c}{ nacional } \\
\cline { 3 - 6 } & & 3 pruebas & 2 pruebas & 1 prueba & 0 pruebas \\
\hline A & 29 & 15 & 7 & 7 & 6 \\
B & 35 & 13 & 7 & 3 & 8 \\
C & 30 & 5 & 9 & 2 & 29 \\
D & 34 & 0 & 3 & $\mathbf{1 3}$ & $\mathbf{5 6}$ \\
\hline Total de escuelas & $\mathbf{1 2 8}$ & $\mathbf{3 3}$ & $\mathbf{2 6}$ & & \\
\hline
\end{tabular}

En el conglomerado A, que tiene las mejores condiciones socioeconómicas y que lo conformaron 29 escuelas, hay 15 escuelas (un 52\%) que en las tres pruebas aplicadas obtuvieron un promedio por arriba de la media nacional, mientras que seis escuelas $(21 \%)$ mostraron valores por debajo de la media nacional.

En el conglomerado B, que está en segundo lugar en cuanto a las condiciones socioeconómicas, de las 35 escuelas que lo conforman 13 (37\%) exhibieron un promedio por arriba de la media en las tres pruebas, mientras que ocho escuelas (23\%) obtuvieron un promedio por debajo de la media nacional en las tres pruebas realizadas por el TERCE.

En el conglomerado $\mathrm{C}$, que está en tercer lugar en cuanto a las condiciones socioeconómicas y conformado por 30 escuelas, cinco de ellas (17\%) obtuvieron un promedio por arriba de la media nacional en las tres pruebas, mientras que 13 escuelas (43\%) obtuvieron en las pruebas del TERCE promedios por debajo de la media nacional (Tabla 4).

El cuarto conglomerado, denominado con código arbitrario D, tiene las condiciones socioeconómicas más desfavorecidas (conformado por 34 escuelas). Sólo tres escuelas (un 9\%) obtuvieron puntajes por arriba de la media nacional en dos pruebas, mientras que $29(85 \%)$ escuelas obtuvieron puntajes promedio por debajo de la media nacional.

\section{Intervalos de confianza de los puntajes promedios de las pruebas por conglomerados}

\section{Para tercer grado}

En la Figura 1 se muestran los intervalos de confianza para la media aritmética por conglomerado obtenido. En la prueba de lectura de tercer grado, en el conglomerado A, el intervalo de confianza fue de $682 \pm 1.96 * 6.64$; en el conglomerado B fue de $672 \pm 1.96 * 4.56$; en el conglomerado $C$ fue de $653 \pm 1.96 * 7$; y, finalmente, en el conglomerado D fue de $613 \pm 1.96 * 9.75$.
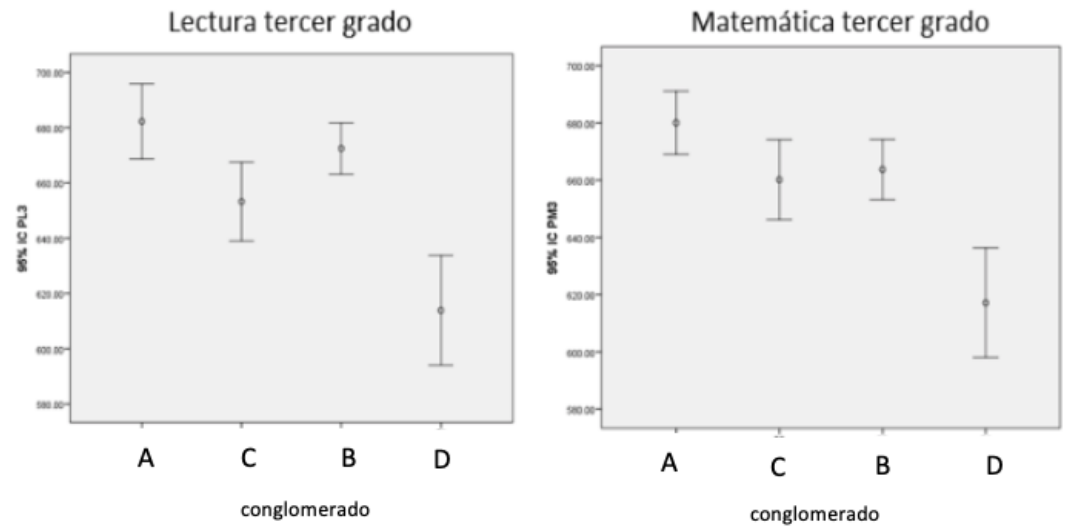
Figura 1. Intervalos de confianza de los puntajes promedios de las pruebas de tercer grado según conglomerado, en el TERCE Panamá.

En la prueba de matemática de tercero, en el conglomerado A el intervalo de confianza fue de 680 $\pm 1.96 * 5.39$; en el conglomerado B fue $663 \pm 1.96 * 5.18$; en el conglomerado C fue de $660 \pm 1.96 * 6.86$; y finalmente, en el conglomerado D fue de $617 \pm 1.96 * 9.43$ (Figura 1 ).

Es posible también observar que en ambas pruebas, el menor promedio se detecta en el conglomerado D que es el grupo de escuelas panameñas en donde los estudiantes tienen condiciones socioeconómicas más desfavorables.

\section{Para sexto grado}

Por otro lado, en la Figura 2 se puede apreciar que en la prueba TERCE de lectura, en el conglomerado A el intervalo de confianza fue de $690 \pm 1.96 * 5.54$; en el conglomerado B fue $672 \pm 1.96 * 5.73$; en el conglomerado

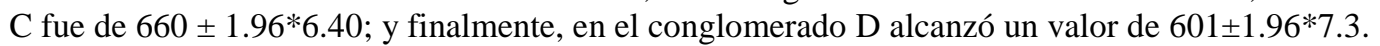

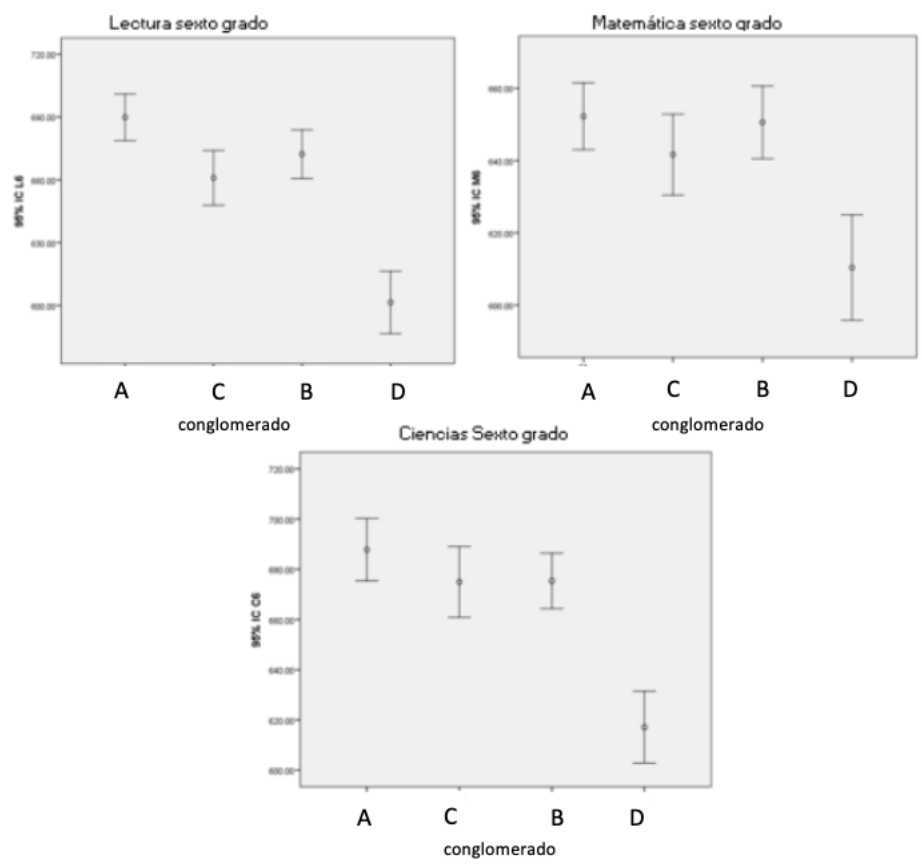

Figura 2. Intervalos de confianza de los puntajes promedios de las pruebas de sexto grado según conglomerado en el TERCE Panamá.

En la prueba TERCE de ciencias, en el conglomerado A el intervalo de confianza fue $687 \pm 1.96 * 6.07$; en el conglomerado B fue $675 \pm 1.96 * 5.42$; en el conglomerado C fue $674 \pm 1.96 * 6.90$; y, en el conglomerado D fue $617 \pm 1.96 * 7.03$. Finalmente, en la prueba TERCE de matemática, en el conglomerado A el intervalo de confianza fue $652 \pm 1.96 * 4.51$; en el conglomerado B fue $650 \pm 1.96 * 4.96$; en el conglomerado C fue $641 \pm$ 1.96*5.49; y, en el conglomerado D fue de $610 \pm 1.96 * 7.16$, tal como se observa en la Figura 2.

\section{Caracterización sociodemográfica de los conglomerados}

\section{Nivel socioeconómico}

En la Figura 3 se muestra la variabilidad del índice de nivel socioeconómico para los diferentes conglomerados por grados escolares en este estudio, en el contexto del TERCE Panamá. Se observa que el conglomerado A 
está posicionado hacia los valores más altos, seguido del conglomerado B. Le siguen el conglomerado C y el $\mathrm{D}$, que exhiben valores menores. Esta instancia aplica tanto para tercer como para sexto grado de las escuelas panameñas bajo estudio.
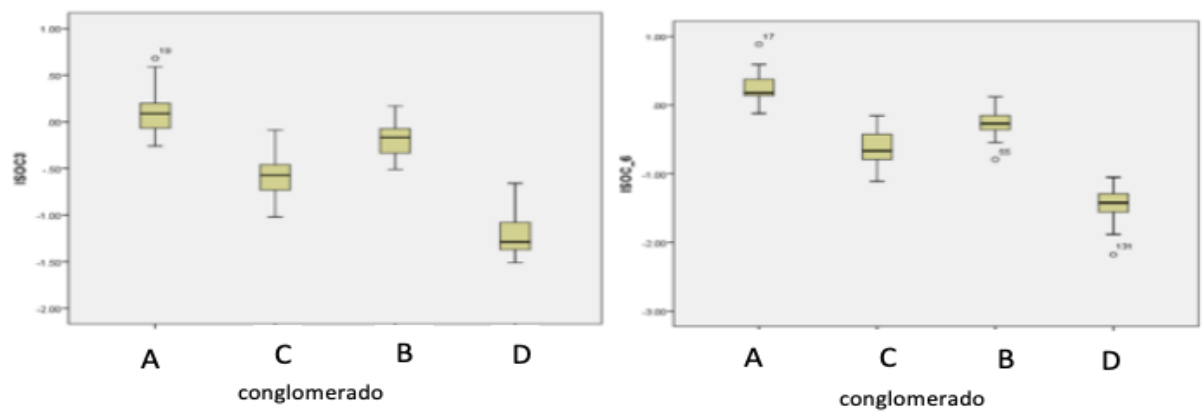

Figura 3. Estadísticas descriptivas del nivel socioeconómico de los conglomerados generados de los datos del TERCE Panamá.

\section{Educación de la madre y padre de los estudiantes}

Por otra parte, en la Figura 4 se destaca que, en tercer grado, en el conglomerado $D$, que presenta las condiciones socioeconómicas más desfavorables, el porcentaje de madres con ningún grado de estudio o con estudios primarios, alcanzó un valor de 50\%, mientras que el valor para los padres se observa en $47 \%$. En sexto grado en el conglomerado $D$, el porcentaje para la variable que se analiza es de $58 \%$ para madres y padres.

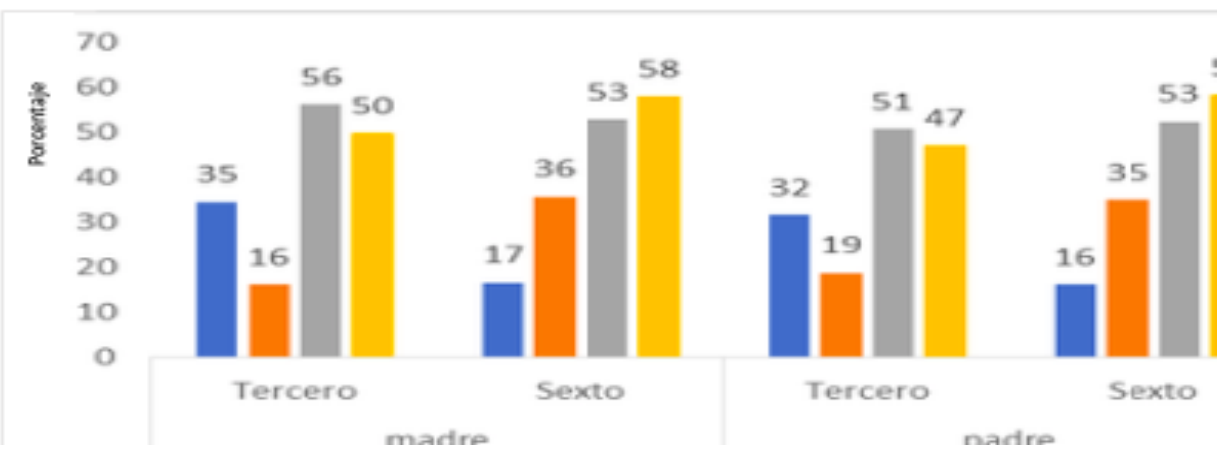

Figura 4. Porcentaje de madres y padres con ningún grado de estudios, o con estudios primarios, por conglomerado según grado del estudiante.

Estos datos corroboran lo que afirman Bourdieu, Inda y Beneitez (2001) quienes indican que "el rendimiento escolar de la acción educativa depende del capital cultural previamente invertido en la familia; y, se olvida además que el rendimiento social y económico de la titulación académica depende del capital social, igualmente heredado, que pueda ser movilizado para respaldarla". 


\section{Trabajo estable pagado de los padres y madres}

Los padres de familia de los estudiantes en tercer grado, que tienen un trabajo estable pagado, alcanzan un valor de 28\%, mientras que en sexto grado, solo un 15\% (Figura 5). La situación de las madres es más difícil, como se observa en la misma figura, pues en tercer grado sólo un $12 \%$ tiene trabajo estable pagado, mientras que en sexto grado es tan solo un valor de 5\%, en el conglomerado D. En este contexto, un estudio reciente de Rodríguez y Guzmán (2019) indica que "en contextos sociales o familiares desfavorecidos es mucho más probable que el estudiante esté expuesto a un conjunto de factores, y no a uno solo, de manera que la exposición combinada o acumulada de éstos es lo que incrementa significativamente la incidencia negativa en el rendimiento".

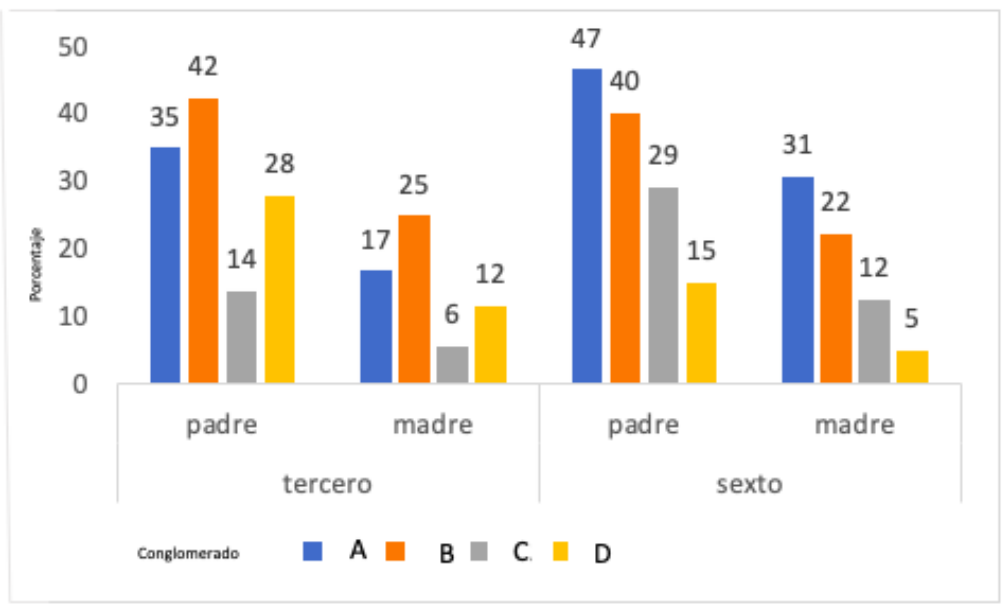

Figura 5. Porcentaje de padres y madres que tienen un trabajo pagado estable por conglomerado según grado del estudiante.

\section{Servicios básicos de la vivienda}

$\mathrm{Al}$ analizar la Figura 6, en el conglomerado D, en sexto grado un 7\% respondió en la prueba en campo no tener servicio de recolección de basura, y en tercero un 6\%; en tercer y sexto grado un $3 \%$ respondieron no tener alcantarillado; en sexto grado un $42 \%$ señaló no tener agua potable y en tercero un $62 \%$. En sexto grado un $20 \%$ señaló no tener luz eléctrica, mientras que en tercer grado fue de $18 \%$. Además, se aprecia que el conglomerado D tiene el mayor porcentaje de carencia de agua potable: $61 \%$ y $42 \%$ para tercero y sexto grado, respectivamente; y, en luz eléctrica un $18 \%$ y $20 \%$ para tercero y sexto grados. 


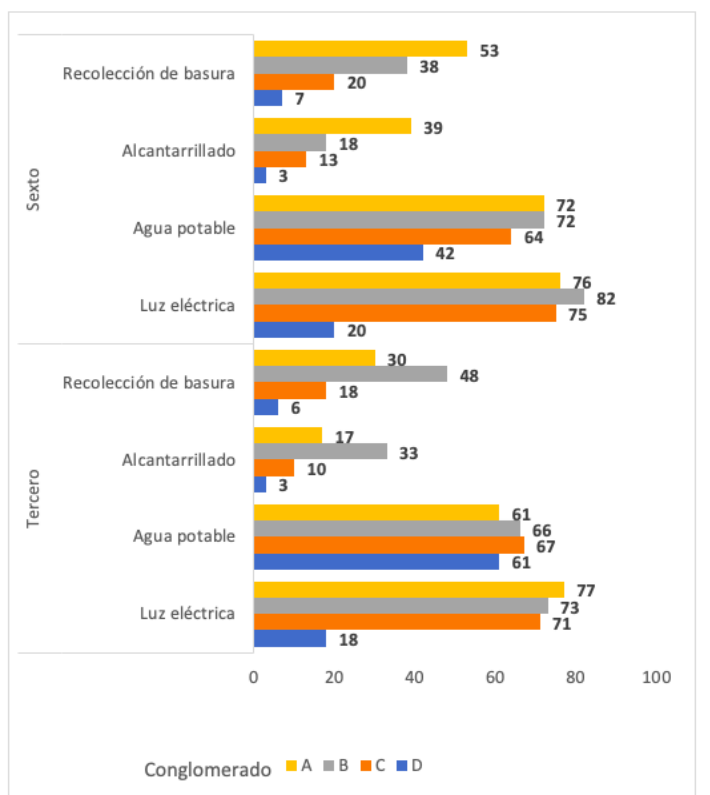

Figura 6. Porcentaje de viviendas que cuentan con servicios básicos por conglomerados según estudiantes por grado.

Esta instancia es afín a la descrita por el Fondo de las Naciones Unidas para la Infancia (UNICEF, 2018), que se refiere a estos mismos servicios. La misma entidad indica además que una de las enfermedades producidas por falta de agua y saneamiento es la diarrea, y afecta a los niños, ocasionando en alta proporción incluso la muerte. En casos de niños con cuadros repetitivos de diarrea, quedan vulnerables a la desnutrición (la diarrea es un problema de salud pública producida por la falta de agua y saneamiento, como lo confirma esta fuente consultada). Estas instancias de salud pública, reflejadas también en los datos del presente estudio, tienen repercusión directa en asistencia a la escuela y aprendizaje efectivo.

\section{Expectativas de los padres en cuanto al nivel educativo más alto que alcanzarán sus hijos}

Según los padres de familia, al indicar cuál es el nivel de educación más alto que lograrán sus hijos, en el conglomerado D en tercer grado, un 35\% dijo que secundaria y un 37\% dijo que educación superior. Por otro lado, en sexto grado, un 33\% respondió indicando que llegarán a secundaria y un 18\% a educación superior. Este escenario se muestra en la Figura 7.

Se observa además que, en el conglomerado $\mathrm{D}$, las expectativas de los padres de que sus hijos alcancen un grado de secundaria aumentan levemente en un $2 \%$ de tercero a sexto grado y bajan drásticamente de tercero a sexto grado en un 19\% de lograr estudios superiores. Esto está ligado al nivel socioeconómico y cultural de las familias, ya que tienen menos oportunidades de lograr acceso a servicios básicos, vivienda y estabilidad laboral, entre otros aspectos. Al respecto, Intxausti et al. (2014) indican que "la expectativa se refiere a la esperanza de conseguir una cosa si se depara la oportunidad que se desea". En este sentido, la igualdad de oportunidades está muy lejos de ser una realidad: según el Informe PISA (2018), en Panamá el 63\% de la variación en el rendimiento entre centros escolares en la prueba de lectura lo explica el status socioeconómico de los estudiantes. Esto significa que los estudiantes de contextos desfavorecidos tienen menos probabilidades de tener éxito en el sistema educativo en Panamá. 


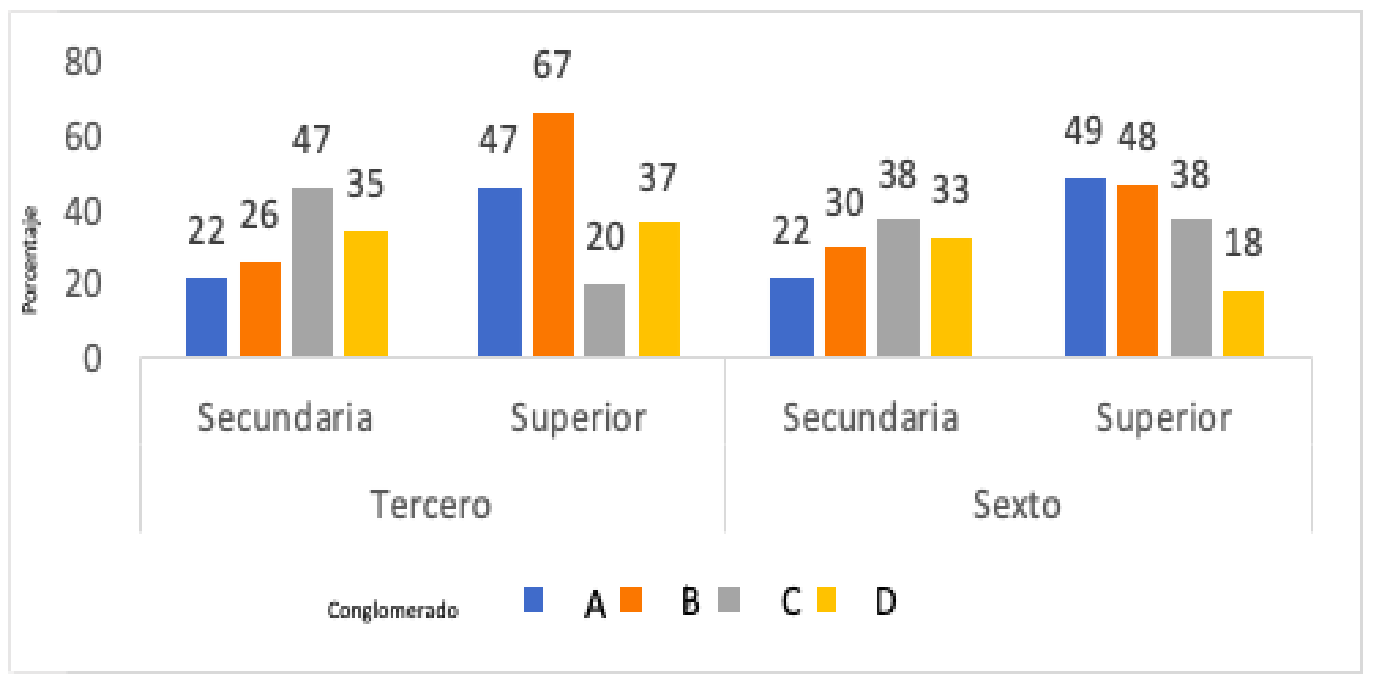

Figura 7. Porcentaje de estudiantes que lograrán terminar la secundaria, o seguir estudios superiores, según expectativa de los padres, por conglomerado y según grado del estudiante, en el TERCE Panamá.

\section{Caracterización de las escuelas por conglomerados}

\section{Cómo accede al puesto el director de escuela}

En el conglomerado D se observó que solo un $20 \%$ del total corresponde a directores nominados por concurso público (Figura 8). Este conglomerado está formado por 34 escuelas; es decir, sólo siete escuelas tienen directores permanentes. Cuando el director no es titular, esto tiene implicaciones, como por ejemplo que el docente asume voluntariamente el cargo sin compromiso de pago a la posición, no tiene las suficientes competencias requeridas al momento para dicho cargo, aprende en el hacer; $\mathrm{y}$, además de dirigir la escuela, en algunos casos, atiende en clase a un grupo de estudiantes (aspectos que también se observaron en un estudio de Jackson et al., 2017). Lo anteriormente expuesto, no le permite al director desarrollar con eficiencia su cargo. En contraste, es positivo que un $70 \%$ de los directores de este conglomerado mostró estudios de licenciatura y maestría, tal como se aprecia en la Figura 8, aunque este porcentaje es mucho menor en los conglomerados A y B, que corresponden a un nivel socioeconómico superior.

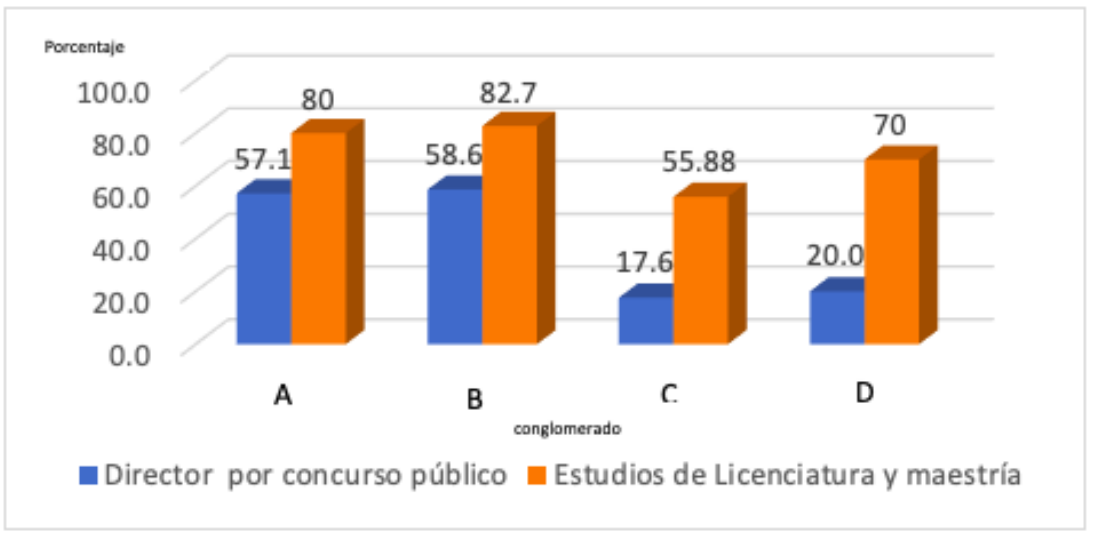

Figura 8. Porcentaje de directores (datos expresados según como se accede al cargo). 


\section{Facilidades con las que cuenta la escuela}

Según los datos analizados, en las escuelas del conglomerado D, ninguna tiene salón de artes o de música; un $10 \%$ dispone de un laboratorio de ciencias. El 53\% tiene cancha deportiva, mientras que un $70 \%$ dispone de una sala de computación, tal como se aprecia en la Figura 9.

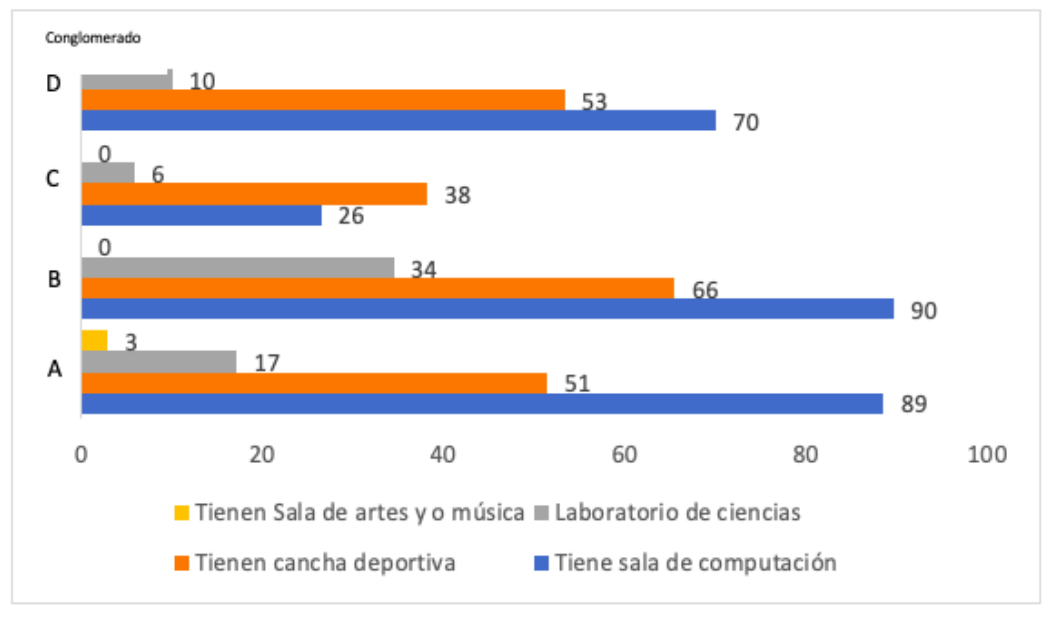

Figura 9. Porcentaje de escuelas que cuentan con canchas deportivas, laboratorios y salas de computación, por conglomerado.

\section{Conglomerado con características socioeconómicas desfavorables, con escuelas con promedio por arriba del promedio nacional}

En el conglomerado D, donde las condiciones socioeconómicas desfavorables tienen mayor severidad en tercer grado, se encontraron cuatro escuelas con promedio por arriba del promedio nacional, mientras que en sexto grado no se encontró ninguna escuela.

De igual modo, luego del análisis estadístico realizado en este estudio, en el conglomerado C (donde las condiciones socioeconómicas desfavorables tienen menos severidad que en el D), se encontraron cinco escuelas en tercer grado y otras cinco escuelas en sexto grado por arriba de la media.

\section{Características de madres y padres de familia}

Con base en las figuras analizadas en el conglomerado $\mathrm{C}$, en el cual se encuentran las tres escuelas que obtuvieron un promedio superior al promedio nacional (en las pruebas de español, matemática y ciencias), los hogares de los estudiantes se caracterizan de la siguiente manera: un alto porcentaje de madres solo tiene estudios primarios, en tercer grado $56 \%$ y en sexto grado $53 \%$. Los padres tienen porcentajes parecidos, $51 \%$ en tercero y $53 \%$ en sexto, respectivamente. Tienen además trabajo estable pagado: un $14 \%$ de los padres de tercer grado y un $29 \%$ de los padres de sexto grado. En las madres los porcentajes son menores, $6 \%$ en tercero y $12 \%$ en sexto.

\section{Características de las viviendas}

En las viviendas de los estudiantes de tercer grado, del conglomerado C, un $71 \%$ tiene luz eléctrica, un $67 \%$ tiene agua potable, un $10 \%$ tiene alcantarillado y un $18 \%$ cuenta con servicio de recolección de basura. En cuanto a las viviendas de estudiantes de sexto grado del conglomerado C, el $75 \%$ tiene luz eléctrica, el $64 \%$ agua potable, el $13 \%$ tiene alcantarillado y el $20 \%$ cuenta con servicio de recolección de basura (estos valores se mostraron en la Figura 6). 
En cuanto a servicios de comunicación que cuentan los estudiantes de tercero y sexto grado en sus viviendas, en promedio son: el $9 \%$ posee televisión por cable y solo un 5\% cuenta con conexión a Internet.

En relación con los electrodomésticos que se disponen en la vivienda, los estudiantes de tercero y sexto grado, en promedio, presentan los siguientes valores: el 64\% tiene celular con acceso a Internet en la vivienda, el $42 \%$ tiene celular sin acceso a internet, el 51\% lavadora, el 51\% tiene refrigeradora, un 65\% computadora, el 33\% televisión, y finalmente el $37 \%$ posee radio o equipo de música.

El 70\% de los estudiantes de tercero y sexto grado en sus viviendas no tienen ningún vehículo a motor en sus viviendas, por los datos analizados.

\section{Expectativas de los padres de familia}

Según las expectativas de los padres, de los estudiantes de tercer grado, el $47 \%$ manifiesta que sus hijos terminarán la secundaria, mientras que un $20 \%$ indicó que terminarán la educación superior. Los padres de los estudiantes de sexto grado piensan que el 38\% terminarán la educación secundaria y un 38\% indicó que terminarán la educación superior.

\section{Características de las escuelas}

En las escuelas del conglomerado C, sólo el $17.6 \%$ de ellas tiene un director que ocupa su puesto por concurso. Es decir que de las 30 escuelas, aproximadamente cinco tienen un director por concurso. En cuanto a las facilidades con las que cuentan, el 38\% de las escuelas tiene canchas deportivas, el $26 \%$ tiene sala con computadoras (sin Internet) para uso de los estudiantes. Ninguna escuela de este conglomerado dispone de una sala de arte, o de música, ni laboratorio de ciencias.

\section{Discusión}

En términos generales, los puntajes de las pruebas TERCE para el país fueron significativamente menores al promedio latinoamericano de dicha evaluación. Al analizar los puntajes según conglomerados, los puntajes de las pruebas para los estudiantes de los conglomerados $\mathrm{C}$ y $\mathrm{D}$ fueron significativamente menores al promedio de los conglomerados A y B, y significativamente menores al promedio de la evaluación del TERCE. Esto evidencia diferencias debido a la condición socioeconómica.

Es posible atribuir esta diferencia de puntajes a dos factores: uno es la familia y el otro factor es el maestro (término aplicado en Panamá a los profesores de una escuela dada). En este sentido, es adecuado destacar que en las escuelas oficiales agrupadas en los conglomerados de nivel socioeconómico más bajo (que en el caso del presente estudio es el conglomerado $C^{9}$ ), cerca de un $97 \%$ de las familias de los estudiantes son de áreas rurales (en muchos de los casos son áreas de difícil acceso). Adicionalmente, en el conglomerado $\mathrm{D}^{10}$, alrededor de un $50 \%$ de las familias proviene de pueblos originarios, esto es, de las comarcas Guna, Emberá Wounaan y Ngäbe Buglé.

En este contexto, el capital social y cultural de la familia juega un papel muy importante en el ámbito escolar. Sobre esto hay numerosos estudios que lo evidencian, como, por ejemplo: (i) las expectativas que tenga la familia para que sus hijos logren alcanzar estudios superiores, aumenta los rendimientos en el aprendizaje (Méndez y Ramírez, 2011); y, (ii) el apoyo y motivación que los padres dan a sus hijos, lo cual ayuda a que éstos tengan un mejor aprovechamiento del aprendizaje (Beneyto, 2015). Es por ello que la importancia de generar estudios que indaguen sobre el capital social familiar y sus repercusiones en el aprendizaje es sumamente clave.

\footnotetext{
${ }^{9}$ El conglomerado C lo forman 30 escuelas; sólo una escuela es de área urbana.

${ }^{10}$ El conglomerado D lo forman 34 escuelas; 15 de ellas son de áreas comarcales; en las restantes escuelas, que no están clasificadas como comarcales (es decir dentro de la jurisdicción de una comarca indígena), existe un número significativo de presencia de estudiantes de pueblos originarios.
} 
Con este panorama desalentador identificado a través de este estudio, habrá que interpretar con cautela los resultados de las pruebas de lectura, matemática y ciencias, ya que hay un porcentaje elevado (alrededor de un $50 \%$ de escuelas, que se agrupan en el conglomerado D), que tiene el nivel socioeconómico más bajo y representa a áreas comarcales de Panamá. Esto significa que su lengua materna es el Ngäbe, el Emberá o el Guna. Teniendo en cuenta esto, se cita a continuación un aspecto sumamente importante que manifiesta Schmelkes (2018):

"Existe un vacío en la información que ofrecen estas pruebas estandarizadas, pues no es posible determinar si los bajos resultados de los alumnos indígenas se deben a que no entienden la instrucción en español, o porque no poseen el conocimiento; tampoco se mide qué saben de su lengua. Sigue diciendo: "No estamos diciendo gran cosa acerca de lo que saben los alumnos indígenas, incluso en Español. Eso es muy importante porque estos niños aprendieron el Español como una segunda lengua y es algo que no se está considerando en las pruebas que estamos haciendo nosotros. Tampoco de Matemática sabemos si el problema de no responder es porque no saben Matemáticas o porque no entienden el Español de la prueba", agregó. Tenemos un problema muy serio que nos lleva a la conclusión de que los niños indígenas están reprobados, lo cual es una conclusión que me parece totalmente falsa. Más bien lo que tendríamos que concluir es que no podemos decir cuánto saben los alumnos indígenas en Lenguaje y Comunicación y en Matemáticas, aunque sabemos que ahí hay un problema y un rezago que tenemos que atender. Es muy difícil intentar con pruebas estandarizadas evaluar lo que saben los niños de la lengua indígena”, subrayó la autora.

Por otro lado, Treviño (2006) indica que "los resultados de la población indígena en estos exámenes deberían conllevar interpretaciones distintas a las que se darían para otras poblaciones. Es necesario, entonces, calificar cuidadosamente los resultados de las poblaciones indígenas en estos exámenes, y queda a la investigación la tarea de desentrañar los efectos de los factores culturales y los constructos que mide el examen. Por lo expuesto, el equipo investigador considera que es necesario realizar estudios adicionales más profundos y puntuales, teniendo como marco de referencia aspectos socioculturales de las áreas comarcales e instrumentos que midan, por ejemplo, el español como segunda lengua, entre otros aspectos a evaluar.

Otro tema importante para mencionar es la formación del maestro de educación bilingüe intercultural (EBI). En el contexto del presente estudio, en los conglomerados C y D hay una gran presencia de estudiantes de pueblos originarios y esto implica que el maestro debe estar formado en la enseñanza de naturaleza EBI, y debe capacitarse continuamente. Si bien esto ocurre en Panamá, es necesario reforzarlo aún más. En este marco, un artículo interesante es el de Aliata (2017), en el cual se hace una reflexión del trabajo docente y la formación en contextos EBI. Comenta en su artículo que muchos docentes, ya sea provenientes de pueblos originarios, o no, atribuyen el fracaso a los estudiantes y sus familias. Sin embargo, indagando sobre sus prácticas en el aula, se encontraron falencias en diversas situaciones de aprendizaje (dentro del aula como también a nivel institucional), ya que la metodología tradicional no da respuestas a todas las necesidades y prioridades de enseñanza.

En Panamá, la Universidad Especializada de Las Américas (UDELAS) es quien forma, por designación del Estado, a estos maestros. Adicionalmente, el Ministerio de Educación (MEDUCA) tiene como criterios para nombrar a maestros en áreas comarcales, los siguientes: hablar la lengua originaria, conocer la cultura y obtener la certificación de competencias lingüística y cultural, expedida por la Dirección Nacional de Educación Bilingüe Intercultural. Aún con estos requisitos es necesario que se evalúe (con fines constructivos) a los maestros formados en EBI, el impacto de su enseñanza en el aula y cómo potenciar mayores logros educativos. Asimismo, los recursos didácticos (libros, guías, manuales, entre otros) que se generan para estos contextos deben ser reevaluados (con miras a actualización y mayor pertinencia local). Finalmente, debe indicarse que la supervisión pedagógica es vital y con frecuencia no está presente, en nivel adecuado, en las escuelas.

Por otra parte, el perfil del docente juega un papel importante en la selección de estos maestros. Hay mucho camino que recorrer en la enseñanza EBI y si no se da un giro de $180^{\circ}$, poco se podrá hacer para lograr aprendizajes en los estudiantes de áreas más vulnerables. En este sentido, la reflexión de Muñoz (citado en INEE, 2007), dice que la fuerza que existe de la relación entre el capital social y el rendimiento académico no es tal, sino que los procesos educativos e insumos no se adaptan a las características de las distintas poblaciones de los estudiantes. Esto significa que dichos procesos no son ni cultural ni pedagógicamente pertinentes, y que por lo mismo requieren de revisión y mejora continua. 
Finalmente, es preocupante ver que en los conglomerados C y D sólo alrededor de un $17 \%$ a $20 \%$ de los directores son asignados por concurso (es decir, son directores titulares). Esto implica que alrededor del $80 \%$ son maestros que fungen como directores encargados bajo el Resuelto No. 257 disponible en la Gaceta de Panamá (MEDUCA, 1979). Dichos maestros no reúnen desde su inicio todas las competencias para liderar un centro escolar, o bien, las generan en periodos de tiempo luego de su designación, con limitaciones de tiempo. El Ministerio de Educación podría abordar este aspecto, que es crucial para las escuelas.

El análisis de conglomerados presentado en este artículo evidencia las diferencias y extremos existentes entre las escuelas analizadas y puede aportar a recomendaciones para el sistema educativo de Panamá. Cerrar la brecha social, económica y educativa es una tarea compleja que requiere voluntad política y trabajo cooperativo de todos los sectores. Basado en estas reflexiones, el equipo de investigadores continuará su línea de investigación sobre la evaluación de la educación, el aprendizaje con pertinencia local, la medición de sus logros, y, en general, su aporte a la sociedad panameña.

\section{Referencias}

Aliata, S. (2017). Reflexiones sobre la figura del docente en contextos interculturales en el Chaco. Educación, lenguaje y sociedad (14), 9.

Bourdieu, P., Inda, A. G., \& Beneitez, M. J. B. (2001). Poder, derecho y clases sociales (Vol. 2). Bilbao: Desclée de Brouwer.

Chaparro, A., González, C. y Caso, J. (2016). Familia y rendimiento académico: configuración de perfiles estudiantiles en secundaria. Revista Electrónica de Investigación Educativa 18(1), 53-68. Recuperado del sitio http://redie.uabc.mx/redie/article/view/774.

División de Evaluación de Logros de Aprendizajes. (2012). Metodología de construcción de grupos socioeconómicos. Agencia de la Calidad de Educación. Santiago de Chile, Chile. Recuperado de http://www.agenciaeducacion.cl/wp- content/uploads/2013/02/Metodologia-de-Construccion-de-GruposSocioeconomicos-SIMCE-2011.pdf.

FAO, UNICEF y WFP. (2018). Panorama de la seguridad alimentaria y nutricional en América Latina y El Caribe - Año 2018. Santiago de Chile, 58-107.

IBM Corp. (2013). IBM SPSS Statistics for Windows, Versión 22.0. (liberada en 2013). Armonk, NY: IBM Corp.

Instituto Nacional para la Evaluación de la Educación. (2007). La educación para poblaciones en contextos vulnerables. Informe anual 2007 (p.135). México. Recuperado de https://www.inee.edu.mx/publicaciones/la-educacion-para-poblaciones-en-contextos-vulnerables-informeanual-2007/.

Intxausti, N., Etxeberria, F. y Joaristi, L. (2014). ¿Coinciden las expectativas escolares de la familia y del profesorado acerca del alumnado de origen inmigrante? Relieve 20 (1), art. 2. DOI: 10.7203/relieve.20.1.3804.

Jackson, D., Estrella, J. y Rodríguez, W. (2017). Enseñando en contextos desfavorables. Estudio de casos de cuatro escuelas oficiales de Panamá. Panamá.

León Jara Almonte, J. y Collahua, Y. (2016). El efecto del nivel socioeconómico en el rendimiento de los estudiantes peruanos: un balance de los últimos 15 años (Vol. 1, pp. 55-108). Grupo de Análisis para el Desarrollo (GRADE).

Ministerio de Educación de Panamá, MEDUCA. (1979). Resuelto No 257. Asignación de funciones. Publicado en la Gaceta Oficial de 24 de febrero de 1979. Panamá.

Ministerio de Educación de Panamá, MEDUCA. (2010). Ley No. 88 “Que reconoce las lenguas y los alfabetos de los pueblos indígenas de Panamá y dicta normas para la Educación Intercultural Bilingüe”. Publicado en la Gaceta Oficial de 22 de noviembre de 2010. Panamá.

Murillo, F. y Hernández-Castilla, R. (2011). Efectos escolares de factores socioafectivos: un estudio multinivel para Iberoamérica. Revista de Investigación Educativa (RIE): 29-2, 407-427. Recuperado (2019, diciembre 12) de

https://www.proceso.com.mx/523357/el-inee-sin-metodo-para-evaluar-aprendizaje-de-alumnos-indigenasconsejera-schmelkes.

PISA (2018). PISA Panamá. Organización para la Cooperación y el Desarrollo Económicos (OCDE). $\begin{array}{llllll}\text { Recuperado } & (2019, & \text { diciembre } & 12) & \text { del } & \text { sitio }\end{array}$ 
MEDUCA http://www.meduca.gob.pa/sites/default/files/WEB/pisa/PISA2018_Panam\%C3\%A1_Resumen Ejecutivo_(1)-compressed.pdf.

Plataforma de Seguridad Alimentaria y Nutricional, SAN. (2018). Recuperado (2019, diciembre 12) de la plataforma oficial en línea: https://plataformacelac.org/storage/app/uploads/public/5e7/ba8/ac3/5e7ba8ac32874961481815.pdf.

Schmelkes, S. (2018). El INEE, sin método para evaluar el aprendizaje de alumnos indígenas: Consejera Schmelkes. Diario Contra Poder, p.9. Recuperado de https://issuu.com/danteoliveyro/docs/contra_1296.

SIMECE. (2016). Decreto Ejecutivo No. 878. El Sistema Integral de Mejoramiento de la Calidad de la Educación, SIMECE (27 de septiembre de 2016).

Tedesco, J. C. (2016). Diez notas sobre los sistemas de evaluación de los aprendizajes. Recuperado (2019, diciembre 12) del sitio: http://repositorio.minedu.gob.pe/handle/123456789/4791.

Treviño, E. (2006). Evaluación del aprendizaje de los estudiantes indígenas en América Latina: desafíos de medición e interpretación en contextos de diversidad cultural y desigualdad social. Revista Mexicana de Investigación Educativa, ISSN 1405-6666, Vol. 11. No. 28, 2006, pp. 225-268.

UNESCO. (2015). Informe de resultados TERCE: logros de aprendizaje. $1^{\text {a }}$. edición. UNESCO (ISBN 97892-3-300041-4).

UNESCO. (2019). El Estudio TERCE 2013. Unesco.org. Recuperado (2019, diciembre 12) de https://es.unesco.org/fieldoffice/santiago/llece/TERCE2013.

\section{Agradecimiento}

Los autores dejan constancia de su agradecimiento a la SENACYT (Secretaría Nacional de Ciencia, Tecnología e Innovación), por el cofinanciamiento recibido para la conducción del estudio en Panamá. El cofinanciamiento fue proporcionado por el proyecto adjudicado y financiado por la Dirección de Aprendizaje de la Ciencia, APR SENACYT Panamá, a través del contrato por mérito CxM No. 153-2016 con código APR-EN-001-16. Se agradece también al Ministerio de Educación de Panamá (MEDUCA), por su oportuna colaboración; a los docentes y estudiantes que apoyaron el estudio; y a la Universidad del Caribe. 